\title{
Differences of temporal dynamics and signal complexity of gamma band oscillations in first-episode psychosis during a working memory task
}

\author{
Pascal Missonnier ${ }^{1,2} \cdot \operatorname{Logos}_{\text {Curtis }^{3}} \cdot$ Joseph Ventura $^{4} \cdot$ François R. Herrmann $^{5}$. \\ Marco C. G. Merlo
}

\begin{abstract}
Gamma band oscillations participate in the temporal binding needed to synchronize cortical networks, involved in early sensory and short term memory processes. In earlier studies, alterations of these neurophysiological parameters have been found in psychotic disorders. To date no study has explored the temporal dynamics and signal complexity of gamma band oscillations in first episode psychosis (FEP). To address this issue, gamma band analysis was performed in 15 FEP patients and 18 healthy controls who successfully performed an adapted 2-back working memory task. Multiple linear and logistic regression models were computed to explore the relationship between the cognitive status and gamma oscillation changes over time. Based on regression model results, phase diagrams were constructed and their complexity was estimated using fractal
\end{abstract}

$\triangle$ Pascal Missonnier

pascal.missonnier@unifr.ch

Unit of Psychiatric Neuroscience and Psychotherapy, Department of Medicine, Faculty of Science, University of Fribourg, Chemin du Musée 5, 1700 Fribourg, Switzerland

2 Mental Health Network Fribourg (RFSM), Sector of Psychiatry and Psychotherapy for Adults, L'Hôpital 140, Case postale 90, 1633 Marsens, Switzerland

3 Young Adult Psychiatry Unit, Division of Specialized Psychiatry, Department of Mental Health and Psychiatry, Faculty of Medicine of the University of Geneva, University Hospitals of Geneva, Rue de Lausanne 67, Genève, 1202 Geneva, Switzerland

4 Department of Psychiatry and Biobehavioral Sciences, UCLA Department of Psychiatry, 300 Medical Plaza, Room 2243, Los Angeles, CA 90095-6968, USA

5 Department of Internal Medicine, Rehabilitation and Geriatrics, University Hospitals of Geneva, 3 chemin PontBochet, Thônex, 1226 Geneva, Switzerland dimension, a mathematical tool that describes shapes as numeric values. When adjusted for gamma values at time lags -3 to $-4 \mathrm{~ms}$ and -15 to $-16 \mathrm{~ms}$, FEP patients displayed significantly higher time-dependent changes than controls, independently of the nature of the task. The present results are consistent with a discoordination of the activity of cortical generators engaged by the stimulus apparition in FEP patients, leading to a global binding deficit. In addition, fractal analysis showing higher complexity of gamma signal, confirmed this deficit. Our results provide evidence for recruitment of supplementary cortical generators as compensating mechanisms and yield further understanding for the pathophysiology cognitive impairments in FEP.

Keywords First-episode psychosis · Gamma band oscillations - Temporal dynamics · Fractal dimension . Cortical binding · Working memory

\section{Introduction}

Schizophrenia is a severe psychiatric disorder characterized by delusions, hallucinations and disorganization of thought, as well as impairments of social functioning (Insel 2010; Kahn et al. 2015). Along with these clinical symptoms, cognitive impairments represent a core feature of the pathology (Reichenberg et al. 2009; Insel 2010; Kahn et al. 2015). These cognitive impairments seem to be related to a deficit of activation of neural networks (Pittman-Polletta et al. 2015). Indeed, anatomical and functional investigations using imaging methods (MRI/fMRI) revealed dysfunctional thalamocortical interactions in schizophrenia (Shenton et al. 2001; Glahn et al. 2008; Welsh et al. 2010; Woodward et al. 2012; Wagner et al. 2013; Woodward 2016). Discordant data from the MRI literature 
demonstrated that changes in brain functioning may be characterized by either abnormally reduced, or increased interactions in extended frontal and parietal cortical circuits, respectively (Woodward et al. 2012). These impaired interactions between brain regions support the notion of a disconnectivity syndrome in schizophrenia, which suggests dysfunctional coordination or synchronization of neuronal oscillations in large-scale networks (Uhlhaas 2013). Alterations of neuronal synchrony parameters of these brain regions have also been demonstrated to be implicated in the pathophysiological processes of the disorder (Uhlhaas et al. 2008; Roach et al. 2013; Skåtun et al. 2016).

The precise synchronization of distributed neuronal activities can reflect the activity of a selective assembly of neurons into functional groups, which are needed for the temporal coding that supports fast selection and multiregional cortical binding (Singer 2001; Varela et al. 2001). Large bodies of evidence pointed towards the role of high oscillatory activities like oscillations in the gamma band $(>40 \mathrm{~Hz})$ in this integrative mechanism (Roach and Mathalon 2008; Basar and Guntekin 2013). Previous contributions documented the presence of significant changes in event-related gamma-band oscillation parameters in schizophrenia during different experimental sensory-independent tasks (Spencer et al. 2004, 2008). Similar neurophysiological gamma oscillations profiles were reported in first-episode psychosis (FEP), as well as schizophrenia patients (Leicht et al. 2015; Basar 2013; Symond et al. 2005). Earlier reports demonstrated a reduction of phase and dysfunctional long-range synchrony during various cognitive paradigms (Pittman-Polletta et al. 2015; for review, Uhlhaas and Singer 2011, 2012; Basar 2013), thus highlighting a deficit in sensory processing in psychotic patients. Moreover, a global decrease, as well as a latency increase, of gamma synchrony in initial phases of encoding ( $<150 \mathrm{~ms}$ post-stimulus) were also described for relevant stimuli in a conventional oddball paradigm in FEP (Lee et al. 2003; Perez et al. 2013). Such findings provide additional evidence of a core disturbance of connectivity across distributed neuronal circuits, which impair the perceptual and cognitive processing of target stimuli in FEP patients. Altogether, the abnormal early gamma synchronization raises the question of the link between connectivity and temporal relation of distributed neural responses to form functional circuits in this population of psychiatric patients. Finally, even though gamma band oscillations play a key role in the synchronization of the cortical networks involved in early sensory processing, these fast oscillations may also be modulated in tasks involving working memory in schizophrenia patients (Basar-Eroglu et al. 2007).

From a neurophysiological point of view, appropriate synchronization depends on the ability of neural networks to self-organize and form stable electroencephalography (EEG) patterns at the initial stages of cognitive activation (Stam 2005). Moreover, an organized neural network includes a temporal memory so that its current state (time $n$ ) may be predicted based on an earlier one (time lag $n-1 \mathrm{~ms}$ ). According to this conceptualization, it is crucial to investigate this temporal evolution of the gamma oscillations.

To date, no study has examined temporal changes in gamma band dynamics in first-episode psychosis. In particular, it has not been established whether specific cognitive deficits may be associated to deficits of gamma synchrony at specific time lags of the temporal evolution of these oscillations. We hypothesized that temporal synchronization abnormalities of gamma oscillations may occur in the 1-20 ms time-range following stimulus onset, which is the time of cortical binding. We also expected to find a higher intricacy of the gamma signal. In patients with FEP, such abnormalities may thus participate in the generation of the global binding deficit linked to the development of the psychosis. Therefore, we performed an EEG activation study associated with time lag and fractal dimension analysis of gamma oscillations in healthy controls and FEP patients that successfully performed an $n$ back working memory (WM) task, as well as oddball detection and passive fixation tasks.

\section{Subjects and methods}

\section{Participants}

Fifteen patients with first-episode psychosis (12 men, 3 women; mean age \pm SD $21.9 \pm 2.6$ years, age-range 18-26 years) were recruited in the specialized program for young adult psychiatric patients (JADE) in Geneva, Switzerland. At first contact with mental health services, patients were assessed for positive psychotic symptoms (defined as hallucinations, delusions or formal thought disorders). The diagnosis was made according to the DSMIV criteria (American Psychiatric Association. 1994) by two experienced psychiatrists who were independent of the study. Six patients were diagnosed with schizophreniform psychotic disorder and nine with schizophrenia. Five patients had a history of recreational cannabis use. Patients with other types of drug abuse were excluded, as well as with history of sustained head injury or other neurologic or other psychiatric disorders. The clinical and pharmacologic characteristics of the patients are summarized in Table 1.

Patients were clinically rated for symptom severity using items of the Brief Psychiatric Rating Scale (BPRS) (Ventura et al. 1993). Consistent with Ventura and colleagues (Ventura et al. 2000), we used a total score of 
Table 1 Clinical and pharmacological characteristics of patients with first-episode psychosis (age $21.9 \pm 2.6$ years old)

\begin{tabular}{ll}
\hline Delay from first contact to EEG (days) & $57.0 \pm 16.5$ \\
Duration of untreated psychosis (month) & $10.5 \pm 1.4$ \\
Positive symptoms (BPRS subscales) & \\
Bizarre behavior & $1.6 \pm 0.9$ \\
Unusual thought content & $2.5 \pm 1.3$ \\
Hallucination & $1.7 \pm 1.4$ \\
Suspiciousness & $3.1 \pm 1.4$ \\
Negative symptoms (BPRS subscales) & \\
Blunted affect & $3.2 \pm 1.2$ \\
Emotional withdrawal & $1.9 \pm 1.5$ \\
CPZ equivalents, mg/day & $262 \pm 202$ \\
\hline
\end{tabular}

Data represent mean $\pm \mathrm{SD}$

BPRS Brief Psychiatric Rating Scale, CPZ Chlorpromazine, $m g$ milligrams

negative (blunted affect and emotional withdrawal) and positive (bizarre behavior, unusual thought content, hallucinations and suspiciousness items) symptoms items. A group of 18 healthy controls without history of sustained head injury or other neurologic or psychiatric disorders was recruited (9 men, 9 women; mean age \pm SD $24.4 \pm 1.5$ years; age range 22-27 years). All participants had normal or corrected-to-normal visual acuity.

After careful assessment of patients' ability to understand the proposed project, we obtained informed written consent from all patients before final inclusion in the study. Controls also provided informed consent. The study was approved by the Ethics Committee of the University Hospitals of Geneva, Switzerland, and the study protocol was conformed to the Helsinki Declaration.

\section{Experimental design}

The protocol was previously extensively described in Missonnier et al. (2013). Briefly, participants watched a continuous stream of letters (pseudo-random sequences of consonants and vowels) common to the French alphabet on a computer screen. They were instructed to press a computer-controlled pushbutton as soon as a target appeared (response trials). For non-target stimuli, no motor response was required (no-response trials). Targets were defined according to the oddball (rare event) or to the $N$-back design (see Fig. 1).

At the beginning of the recording session, a passive task was performed: letter series identical to the 2-back task were presented with the instruction to passively watch the series. Each task was tested in sequences composed of 30 images each (including 7 targets).
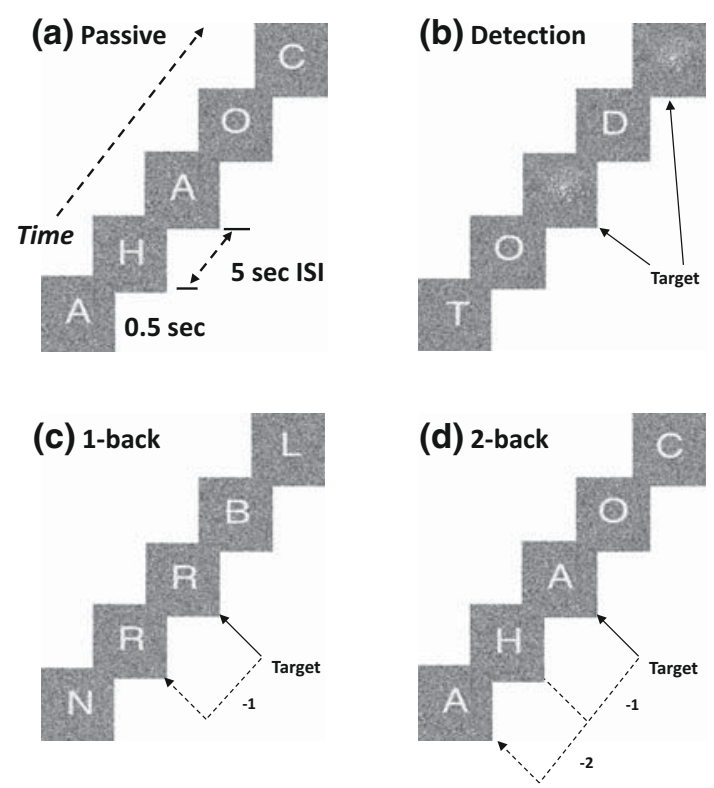

Fig. 1 Schematic representation of the four tasks included in this experimental setting: a in the passive fixation task, letter series identical to the 2-back working memory task are presented, yet the subject must only watch the series; $\mathbf{b}$ in the detection attention task, the subject must press a pushbutton as soon as a background patch without letter is presented; c, $\mathbf{d}$ in the 1-back and 2-back working memory tasks, the subject must press a pushbutton when the letter is identified as being identical to the one presented one trial back (c 1back task) or two trials back (d 2-back task). Stimulus duration $=0.5 \mathrm{~s}$, inter-stimulus interval (ISI) $=5 \mathrm{~s}$

Subjects were kept informed of the nature of the forthcoming task right before each sequence. The protocol started with three sequences of the passive task. Thereafter, a detection task sequence was followed by 1 sequence of the 1-back, 3 sequences of the 2-back, 2 sequences of the 1-back and 2 sequences of the detection task. The duration of the experimental design approximated $40 \mathrm{~min}$.

\section{Electrophysiological recordings}

Continuous EEG (Micromed, Brain Quick system 98, Treviso, Italy) was recorded using 20 surface electrodes according to the 10-20 international electrode placement system (Homan et al. 1987) with linked earlobes as a reference. Skin impedance was kept below $5 \mathrm{k} \Omega$. Electrophysiological signals were sampled at $1024 \mathrm{~Hz}$ with a lower cutoff of $0.33 \mathrm{~Hz}$ and upper cut-off of $120 \mathrm{~Hz}$ (DC amplifiers; Micromed). The electro-oculogram was recorded using two pairs of bipolar electrodes in both vertical and horizontal directions. Single transistor-transistor logic (TTL) pulses synchronized with stimulus onset were recorded and used off-line to segment the continuous EEG data into epochs time-locked to stimulus onset. 


\section{Data processing}

Using BrainVision Analyzer 2 software (Brain Products $\mathrm{GmbH}$, Munich, Germany), EEG signals were corrected for blinks and eye movement artifacts through an independent component analysis (ICA) (Jung et al. 2000). The total analysis window was $4000 \mathrm{~ms}$, starting $500 \mathrm{~ms}$ before stimulus onset. Spatial resolution of EEG data was enhanced by surface Laplacian estimation performed on continuous EEG (regularized 3D spline function, order 4). Such Laplacian calculation acts as a high-pass spatial filter that reduces head volume conduction and cancels out reference electrode influence (Perrin et al. 1987; Babiloni et al. 1996). All subsequent analysis was performed on the Laplacian-transformed EEG signal $\left(\mu \mathrm{V} / \mathrm{m}^{2}\right)$ computed at each electrode. Then, the Laplacian-transformed EEG trials were automatically scanned for contamination by muscular or electrode artifacts (criteria for rejection: voltage step $>50 \mu \mathrm{V} / \mathrm{ms}$ or peak-to-peak deflection within 300-ms intervals $>200 \mu \mathrm{V} / \mathrm{ms}$ ) and the remaining trials were inspected visually to control for residual minor artifacts. To eliminate effects from manual responses and exclude the confounding effect of motor processing, correct answers without motor response (non-target) were analyzed according to the task condition (passive, detection, 1- and 2-back). Then, event-related oscillations (EROs) were averaged over a window of $1050 \mathrm{~ms}$ with a 525-ms prestimulus onset and filtered at 35-45 $\mathrm{Hz}$ (gamma) frequency bands using narrow band-pass filter ( $-48 \mathrm{~dB} /$ octave). Analyses of the segment-evoked oscillations included the frontal (F3, Fz, F4), central (C3, Cz, C4), parietal (P3, Pz, $\mathrm{P} 4)$ and occipital (O1, Oz, O2) electrodes.

\section{Statistical analysis}

EEG data (i.e., value of the gamma fractal dimension) were normalized with a logarithmic transformation to perform parametric one-way repeated comparisons. The normality of data distribution was verified with the Shapiro-Francia test. Statistical analysis was performed to compare EEG measures between groups using a two-way repeated measure ANOVA, with tasks as within-subject factors. The significance values were determined using Bonferroni correction for multiple testing. Post hoc analysis was performed using Fisher's least square difference test (Milliken and Johnson 1994).

Phase space diagrams (Fig. 2) were used to explore the best time lag to discriminate whether neuronal activation was different between controls and FEP. Phase space represents all the possible states over time of a dynamic system. A phase plot thus corresponds to a graph of gamma oscillation values at time $t$ on the $x$-axis versus gamma values at time $t$ minus a lag $(t-\operatorname{lag})$ on the $y$ - axis. Plots were drawn for combined Frontal, Central, Parietal and Occipital electrode sites, task and subject for time lag periods ranging from 1 to $25 \mathrm{~ms}$. Linear regression models (Dwyer and Feinleib 1992), taking into account the clustering of measures within each subject (repeated measure linear regression), were built (Missonnier et al. 2010).

In addition, logistic regression models, which explain the binary psychiatric status (i.e. the group is used as the dependent variable), were built with task, electrode and gamma values at a given time lag $\left(\right.$ gamma $\left._{\text {lag }}\right)$ as the independent variables. Logistic regression models provide odds-ratio to assess the strength of association between presence and absence of psychiatric disorder in a given population.

Fractal dimension is a mathematical tool that allows to precisely discriminate the shape of objects by representing them as a single numeric value. For our results, fractal dimension was thus used to give a measure of the complexity of the phase diagrams. Complexity in our study is interpreted as the higher the numeric value of the fractal dimension, the more intricate and detailed the phase diagrams. Moreover, it can be related to the concept of dynamical complexity, which increases as the level of synchronization between sources in a functional network decreases (Stam 2005). The fractal dimension was estimated using the Fractal analysis software (Fractalyse, CNRS, Bourgogne Franche-Comté University, Version: 2.4; downloadable from http://www.fractalyse.org). Comparison between the two groups was performed using ANOVA while adjusting for task, electrode sites and interactions.

Statistical threshold was set at $p<0.05$. Statistical analyses were performed using the Stata software package (Statcorp, College Station, TX, USA, 2007, release 13.1).

\section{Results}

\section{Repeated measure linear regression of event-related oscillations}

The visual stimuli elicited a series of gamma oscillations in the time range $0-500 \mathrm{~ms}$ for the two groups (Fig. 2a). The assessment of regression coefficients showed that significant group differences in gamma dynamics (i.e. differences in neuronal activity) involved two sets of time lags, i.e. 3-4 ms and 15-16 ms (Table 2). When adjusted for gamma value at lags 3 and $4 \mathrm{~ms}$ in regression models, the gamma oscillations of FEP were significantly different from controls $(t=2.16, \quad p=0.046$ and $t=2.13$, $p=0.048$, respectively) during the interval $0-101 \mathrm{~ms}$ post-stimulus. Similar differences were observed for 
Table 2 Regression coefficients (Column Coeff in $\mu \mathrm{V} / \mathrm{m}^{2}$ ) associated with group effects and their significance at different lag time, determined from repeated measure linear regression models $(N=33$ subjects; see "Subjects and methods" section for details)

\begin{tabular}{|c|c|c|c|c|c|c|c|c|c|c|c|}
\hline \multirow[t]{3}{*}{ Lag } & \multicolumn{11}{|c|}{ Linear regression } \\
\hline & \multicolumn{5}{|c|}{ Group (FEP vs controls) } & \multicolumn{5}{|c|}{ Gamma at time lag } & \multirow[t]{2}{*}{$R^{2}$} \\
\hline & Coeff & $95 \%$ & CI & $t$ & $p$ & Coeff & $95 \%$ & $\mathrm{CI}$ & $t$ & $p$ & \\
\hline 1 & 0.0023 & -0.0001 & 0.0047 & 1.99 & 0.063 & 0.97 & 0.96 & 0.97 & 846.08 & $<0.001$ & 0.93 \\
\hline 2 & 0.0050 & 0.0000 & 0.0101 & 2.11 & 0.050 & 0.87 & 0.86 & 0.88 & 235.01 & $<0.001$ & 0.75 \\
\hline 3 & 0.0075 & 0.0002 & 0.0148 & 2.16 & 0.046 & 0.72 & 0.70 & 0.73 & 80.06 & $<0.001$ & 0.51 \\
\hline 4 & 0.0090 & 0.0001 & 0.0179 & 2.13 & 0.048 & 0.52 & 0.48 & 0.55 & 32.80 & $<0.001$ & 0.26 \\
\hline 5 & 0.0091 & -0.0006 & 0.0188 & 1.99 & 0.063 & 0.28 & 0.24 & 0.33 & 12.78 & $<0.001$ & 0.08 \\
\hline 6 & 0.0078 & -0.0018 & 0.0174 & 1.71 & 0.106 & 0.03 & -0.02 & 0.09 & 1.25 & 0.228 & 0.00 \\
\hline 7 & 0.0054 & -0.0037 & 0.0146 & 1.25 & 0.228 & -0.22 & -0.27 & -0.16 & -7.86 & $<0.001$ & 0.04 \\
\hline 8 & 0.0027 & -0.0058 & 0.0112 & 0.67 & 0.515 & -0.44 & -0.50 & -0.39 & -17.21 & $<0.001$ & 0.18 \\
\hline 9 & 0.0004 & -0.0075 & 0.0082 & 0.10 & 0.922 & -0.64 & -0.68 & -0.59 & -28.26 & $<0.001$ & 0.37 \\
\hline 10 & -0.0009 & -0.0079 & 0.0062 & -0.26 & 0.795 & -0.78 & -0.81 & -0.74 & -42.53 & $<0.001$ & 0.55 \\
\hline 11 & -0.0007 & -0.0068 & 0.0053 & -0.26 & 0.801 & -0.85 & -0.88 & -0.83 & -62.97 & $<0.001$ & 0.66 \\
\hline 12 & 0.0007 & -0.0046 & 0.0060 & 0.28 & 0.782 & -0.87 & -0.89 & -0.85 & -80.42 & $<0.001$ & 0.68 \\
\hline 13 & 0.0030 & -0.0022 & 0.0082 & 1.21 & 0.244 & -0.82 & -0.85 & -0.79 & -56.70 & $<0.001$ & 0.60 \\
\hline 14 & 0.0054 & -0.0005 & 0.0114 & 1.93 & 0.071 & -0.72 & -0.77 & -0.67 & -31.38 & $<0.001$ & 0.46 \\
\hline 15 & 0.0075 & 0.0004 & 0.0145 & 2.24 & 0.039 & -0.58 & -0.65 & -0.51 & -17.56 & $<0.001$ & 0.30 \\
\hline 16 & 0.0086 & 0.0006 & 0.0166 & 2.26 & 0.037 & -0.41 & -0.50 & -0.32 & -9.54 & $<0.001$ & 0.15 \\
\hline 17 & 0.0086 & -0.0002 & 0.0174 & 2.07 & 0.054 & -0.22 & -0.33 & -0.11 & -4.36 & $<0.001$ & 0.04 \\
\hline 18 & 0.0078 & -0.0017 & 0.0173 & 1.73 & 0.101 & -0.04 & -0.15 & 0.08 & -0.65 & 0.527 & 0.00 \\
\hline 19 & 0.0064 & -0.0036 & 0.0165 & 1.35 & 0.196 & 0.13 & 0.01 & 0.26 & 2.34 & 0.032 & 0.02 \\
\hline 20 & 0.0050 & -0.0054 & 0.0155 & 1.02 & 0.324 & 0.28 & 0.16 & 0.39 & 5.04 & $<0.001$ & 0.07 \\
\hline 21 & 0.0040 & -0.0065 & 0.0145 & 0.81 & 0.428 & 0.39 & 0.28 & 0.49 & 7.74 & $<0.001$ & 0.13 \\
\hline 22 & 0.0037 & -0.0065 & 0.0139 & 0.76 & 0.456 & 0.45 & 0.36 & 0.54 & 10.56 & $<0.001$ & 0.17 \\
\hline 23 & 0.0040 & -0.0057 & 0.0138 & 0.88 & 0.393 & 0.48 & 0.40 & 0.56 & 13.02 & $<0.001$ & 0.19 \\
\hline 24 & 0.0049 & -0.0043 & 0.0140 & 1.12 & 0.277 & 0.46 & 0.39 & 0.54 & 13.39 & $<0.001$ & 0.18 \\
\hline 25 & 0.0059 & -0.0028 & 0.0146 & 1.43 & 0.170 & 0.42 & 0.34 & 0.50 & 10.77 & $<0.001$ & 0.14 \\
\hline
\end{tabular}

Each coefficient corresponds to the average difference of the gamma amplitude between first-episode psychotic patients (FEP) and controls. This average difference is adjusted for previous values of gamma at a given time lag (ms), as well as adjusted for age, task, time (range 0-101 ms) and electrodes sites Significant differences are reported in bold $t$ on the $x$-axis and at, respectively, time lags $t-3$ and $t-15$ on the $y$-axis. The elliptical (Fig. 2b) and orbital (Fig. 2c) shapes of the cyclic patterns of gamma dynamics illustrate different neuronal activity at both time lags.

\section{Logistic regression analysis}

When considering the distinction between FEP and controls as a binary dependent variable, multiple (univariate) logistic regression models showed that gamma oscillation values at a given time lag (gamma ${ }_{\mathrm{lag}}$ ) were significantly related to the psychiatric diagnosis. Interestingly, at a given time lag multiple logistic regression analysis confirmed the results obtained with linear regression for gamma values.

During the interval preceding stimulus onset $(-101$ to $0 \mathrm{~ms}$ ), no group difference in gamma dynamics was observed.

\section{Fractal dimension comparisons between controls and FEP}

The distribution of fractal dimensions in controls and patients is illustrated in Fig. 3. For the time lag $3 \mathrm{~ms}$, the fractal dimension was significantly higher in patients (mean $\pm \mathrm{SD}: \quad 0.863 \pm 0.062$ ) compared to controls $[$ mean $\pm \mathrm{SD}: \quad 0.797 \pm 0.050 ; \quad F(1, \quad 496)=10.57$, $p=0.0012]$. Significant task effects $[F(3,496)=5.72$, $p=0.0179]$, as well as an interaction between groups and tasks $[F(3,496)=8.66, p=0.0037]$ were observed. Post hoc analysis revealed that task differences could mainly be explained by differences in the detection condition (Bonferroni $t=2.73, p<0.02$ ). Group differences were also mainly observed in the detection condition (Bonferroni $t=4.39, p<0.001)$. These group differences were still present even after controlling for age and education level. 


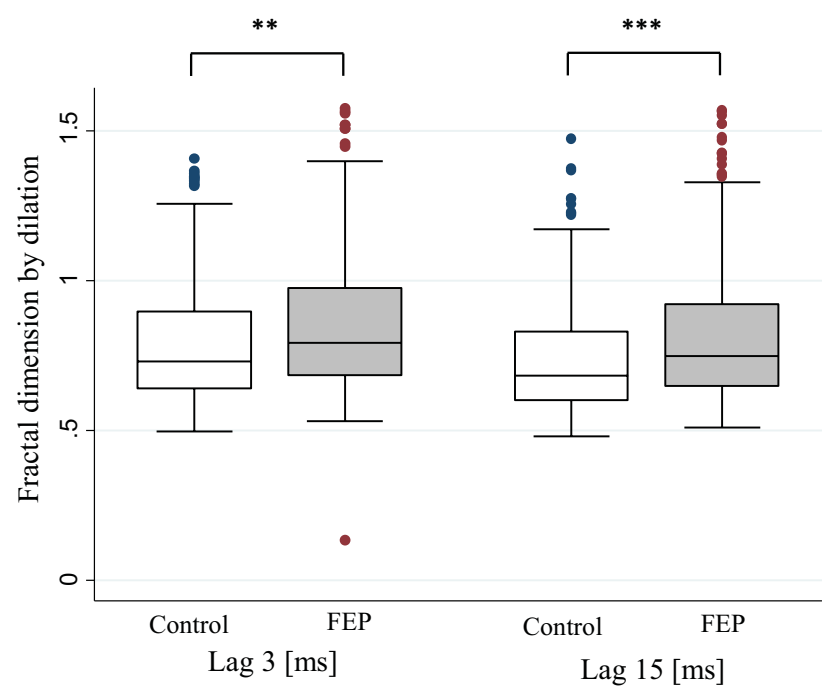

Fig. 3 Box plot of the fractal dimension of phase diagrams for all electrode sites and task conditions at time lag -3 and time lag -15 based on groups (control vs. FEP). Note the highly significant increase of fractal dimension in patients compared to control subjects. See results section for details. $* * p<0.01 ; * * * p<0.001$

For the time lag $15 \mathrm{~ms}$, the fractal dimension of patients with first episode psychosis (mean \pm SD: $0.821 \pm 0.053$ ) was also significantly higher compared to the controls $($ mean $\pm \mathrm{SD}: \quad 0.746 \pm 0.027 ; \quad F(1, \quad 496)=18.03$, $p=0.0001)$.

No other main effects, or two or three-way interactions between factors, were observed for both time lags.

\section{Discussion}

Neuronal oscillations associated with transient communication across brain structures are largely involved as the physiological support of cognitive functioning (Buzsaki and Wang 2012). Alteration of the dynamics of these oscillations is, therefore, associated with cognitive deficits, especially for early sensory and short-term memory processes. Our results show two distinct patterns of fast gamma oscillation abnormalities in FEP. They thus contribute to recent investigations on the pathophysiology of cognitive deficits in schizophrenia, by demonstrating for the first time a clear physiological deficit of cortical binding. Moreover, these differences were related to the temporal dimension of information processing, but not to the difficulty of the cognitive tasks.

Magnetoencephalography investigations indicate that gamma $(35-45 \mathrm{~Hz})$ band activity may represent a neurophysiological correlate to the processing of information (Llinas and Ribary 1993; Ward 2011; Joliot et al. 1994; Ribary 2005; Bertrand and Tallon-Baudry 2000). They supported the idea that stimulus-specific gamma band activity allows to identify early operations activated by the stimulus apparition. In agreement with Singer (2009), we thus included internal timing mechanisms to analyze gamma oscillations. This method allowed us to show significant differences between FEP and controls for two different ranges of lag times. The observed changes in gamma dynamics were neither linked to a motor response, nor task-dependent, but were closely related to stimulus apparition, thus suggesting a deficit in an early-stage of information processing in FEP. In this context, our results are compatible with a disruption of precise temporal coordination from the initial stages of information processing in early psychosis patients and yield additional support to the presence of a sensory integration disorder in this population (Llinas et al. 1999, 2005; Rothenberger 2009).

Our findings of significant differences of gamma band activity in the lag time of $2-4 \mathrm{~ms}$ coincides directly with the thalamocortical conduction time (Albe-Fessard et al. 1986; Allison et al. 1991; Pantev et al. 1991; Ribary et al. 1991). We also demonstrated significant differences for gamma oscillations in the lag time of $15 \mathrm{~ms}$, which refers to perceptual binding occurring in a time segment of $\approx 15$ ms (Joliot et al. 1994; Llinas and Ribary 2001) on the basis of synchronization (i.e. temporal coherence) over the scalp of $40-\mathrm{Hz}$ oscillations. Overall, our results thus clearly demonstrate the presence of both a thalamocortical pathway dysconnectivity, as well as a deficit in cortical binding, respectively, in FEP versus healthy controls. Noteworthy that the reported effects were confirmed using two distinct regression methods both adapted to discriminate between two populations, thereby strengthening the results. Previous physiological studies (Llinas and Ribary 2001; Llinas et al. 1999) revealed that the co-activation of specific and nonspecific thalamocortical loops would be responsible for the synchronization of cortical $40-\mathrm{Hz}$ oscillations, thus generating global binding. In this network, specific thalamic nuclei encode a sensory activity, thereby providing the content of information. Meanwhile, nonspecific thalamic nuclei give rise to the temporal conjunction (i.e., temporal binding of physical attributes of the stimulus) by projecting in a spatially continuous manner to the most superficial layers of all cortical areas (Joliot et al. 1994; Llinas 2003). Cognitively, perceptual organization (Steriade 2006; Uhlhaas et al. 2010; Xing et al. 2012; Buzsaki and Wang 2012) is closely related to sensorial integration. Perception depends on the brain's ability to combine several features of a stimulus into a single object. Therefore, our results strongly suggest synchronization disturbances of the early stimulus processing in FEP patients. In agreement with the literature (Llinas et al. 2005), lag time differences between the two populations were observed on electrode locations covering anterior to posterior brain regions, including 
sensory-motor regions. Altogether, the present results are thus compatible with the hypothesis of a global binding deficit in patients with FEP. Consistent with our findings, several functional imaging studies have revealed alterations in thalamocortical connectivity in schizophrenia (Shenton et al. 2001; Glahn et al. 2008; Welsh et al. 2010; Woodward et al. 2012). Current pathophysiological models of schizophrenia assume synchronization dysfunction at low- and high-frequency ranges between thalamus and cortex, suggesting the presence of a thalamocortical dysrhythmic syndrome in this disorder (Llinas et al. 1999, 2005; Uhlhaas and Singer 2010; Uhlhaas et al. 2013).

Finally, the use of fractal dimension analysis revealed highly significant changes in the temporal evolution of gamma band values, which demonstrate gamma changes of activity in FEP patients. Indeed, the observed average higher value for the FEP group can be interpreted as being close to a dimension 1 (i.e. a straight line); this implies that the phase diagrams were more intricate than the ones described by significantly lower values in the control group.

From a neurophysiological point of view, the higher dynamical complexity of FEP patients reflects an increase in the number of synchronous neuronal networks independently activated in the brain, thereby associated with a poorer level of synchronization (Stam 2005). These findings suggest that FEP patients engaged in a task activated a larger number of cortical generators as soon as the stimulus appeared; yet they did in a non-adapted manner, thus producing an aberrant increased gamma band activity. An increase of gamma band activity was previously reported as being the consequence of a thalamocortical dysrhythmia that generates a cortical continuous theta coherent activity disinhibiting neighboring regions, hence giving rise to an ectopic gamma band activation referred to as the edge effect (Llinas et al. 1999; Steriade 2006). Noteworthy that we previously demonstrated the presence of continuous theta activation in FEP patients (Missonnier et al. 2012). The presence of an edge effect could contribute to the positive symptoms observed in patients, such as verbal hallucinations in the form of voices (Singer 1999; Llinas et al. 2005). Therefore, the inappropriate engagement of large and distributed neural networks found in our study may be likely to represent a compensatory mechanism that integrates incoming sensory stimulus in first episode psychosis patients.

\section{Conclusion and perspectives}

The present data reveal for the first time that both higher variability over time and increased dynamical complexity of the gamma signal are already present in first episode psychosis patients, thus suggesting a role for cortical binding abnormality in the pathophysiology of psychosis.

From a clinical perspective, longitudinal analyses using the same calculation methods are needed to elucidate whether these neurophysiological deficits persist at an advanced stage of the disease, or if such deficits can also be detected before the apparition of characteristic symptoms of the disease (i.e. at the prodromal stage).

From a cognitive standpoint, futures studies are needed to explore the impact of gamma changes on lower frequency oscillations involved in the WM processes in patients with first episode psychosis.

Acknowledgements The authors wish to gratefully thank Prof. A. Prévot for vigilant proofreading of the manuscript.

\section{Compliance with ethical standards}

Conflict of interest This study has not been submitted elsewhere for publication, in whole or in part, and all the authors listed have approved the manuscript. The authors declare that there are no actual or potential conflicts of interest.

\section{References}

Albe-Fessard D, Tasker R, Yamashiro K, Chodakiewitz J, Dostrovsky $\mathrm{J}$ (1986) Comparison in man of short latency averaged evoked potentials recorded in thalamic and scalp hand zones of representation. Electroencephalogr Clin Neurophysiol 65:405-415

Allison T, McCarthy G, Wood CC, Jones SJ (1991) Potentials evoked in human and monkey cerebral cortex by stimulation of the median nerve. A review of scalp and intracranial recordings. Brain 114(Pt 6):2465-2503

Babiloni F, Babiloni C, Carducci F, Fattorini L, Onorati P, Urbano A (1996) Spline Laplacian estimate of EEG potentials over a realistic magnetic resonance-constructed scalp surface model. Electroencephalogr Clin Neurophysiol 98:363-373

Basar E (2013) Brain oscillations in neuropsychiatric disease. Dialogues Clin Neurosci 15:291-300

Basar E, Guntekin B (2013) Review of delta, theta, alpha, beta, and gamma response oscillations in neuropsychiatric disorders. Suppl Clin Neurophysiol 62:303-341

Basar-Eroglu C, Brand A, Hildebrandt H, Karolina Kedzior K, Mathes B, Schmiedt C (2007) Working memory related gamma oscillations in schizophrenia patients. Int $\mathrm{J}$ Psychophysiol 64:39-45

Bertrand O, Tallon-Baudry C (2000) Oscillatory gamma activity in humans: a possible role for object representation. Int $\mathrm{J}$ Psychophysiol 38:211-223

Buzsaki G, Wang XJ (2012) Mechanisms of gamma oscillations. Annu Rev Neurosci 35:203-225

Dwyer J, Feinleib M (1992) Introduction to statistical models for longitudinal observation. In: Dwyer $\mathrm{JH}$ et al (eds) Statistical models for longitudinal studies of health. Oxford University Press, New York, pp 3-47

Glahn DC, Laird AR, Ellison-Wright I, Thelen SM, Robinson JL, Lancaster JL, Bullmore E, Fox PT (2008) Meta-analysis of gray matter anomalies in schizophrenia: application of anatomic likelihood estimation and network analysis. Biol Psychiatry 64:774-781 
Homan RW, Herman J, Purdy P (1987) Cerebral location of international 10-20 system electrode placement. Electroencephalogr Clin Neurophysiol 66:376-382

Insel TR (2010) Rethinking schizophrenia. Nature 468:187-193

Joliot M, Ribary U, Llinas R (1994) Human oscillatory brain activity near $40 \mathrm{~Hz}$ coexists with cognitive temporal binding. Proc Natl Acad Sci USA 91:11748-11751

Jung TP, Makeig S, Humphries C, Lee TW, McKeown MJ, Iragui V, Sejnowski TJ (2000) Removing electroencephalographic artifacts by blind source separation. Psychophysiology 37:163-178

Kahn RS, Sommer IE, Murray RM, Meyer-Lindenberg A, Weinberger DR, Cannon TD, O'Donovan M, Correll CU, Kane JM, van Os J, Insel TR (2015) Schizophrenia. Nat Rev Dis Prim $1: 15067$

Lee KH, Williams LM, Haig A, Gordon E (2003) "Gamma (40 Hz) phase synchronicity" and symptom dimensions in schizophrenia. Cogn Neuropsychiatry 8:57-71

Leicht G, Andreou C, Polomac N, Lanig C, Schöttle D, Lambert M, Mulert C (2015) Reduced auditory evoked gamma band response and cognitive processing deficits in first episode schizophrenia. World J Biol Psychiatry 16:1-16

Llinas R (2003) Consciousness and the thalamocortical loop. Int Congr Ser 1250:409-416

Llinas R, Ribary U (1993) Coherent 40-Hz oscillation characterizes dream state in humans. Proc Natl Acad Sci USA 90:2078-2081

Llinas R, Ribary U (2001) Consciousness and the brain. The thalamocortical dialogue in health and disease. Ann N Y Acad Sci 929:166-175

Llinas RR, Ribary U, Jeanmonod D, Kronberg E, Mitra PP (1999) Thalamocortical dysrhythmia: a neurological and neuropsychiatric syndrome characterized by magnetoencephalography. Proc Natl Acad Sci USA 96:15222-15227

Llinas R, Urbano FJ, Leznik E, Ramirez RR, van Marle HJ (2005) Rhythmic and dysrhythmic thalamocortical dynamics: GABA systems and the edge effect. Trends Neurosci 28:325-333

Milliken GA, Johnson DE (1994) Analysis of messy data, Vol I: Designed experiments. Chapman and Hall/CRC Press, Boca Raton, FL

Missonnier P, Herrmann FR, Michon A, Fazio-Costa L, Gold G, Giannakopoulos P (2010) Early disturbances of gamma band dynamics in mild cognitive impairment. J Neural Transm 117:489-498

Missonnier P, Herrmann FR, Zanello A, Badan Ba M, Curtis L, Canovas D, Chantraine F, Richiardi J, Giannakopoulos P, Merlo MC (2012) Event-related potentials and changes of brain rhythm oscillations during working memory activation in patients with first-episode psychosis. J Psychiatry Neurosci 37:95-105

Missonnier P, Hasler R, Perroud N, Herrmann FR, Millet P, Richiardi J, Malafosse A, Giannakopoulos P, Baud P (2013) EEG anomalies in adult ADHD subjects performing a working memory task. Neuroscience 241:135-146

Pantev C, Makeig S, Hoke M, Galambos R, Hampson S, Gallen C (1991) Human auditory evoked gamma-band magnetic fields. Proc Natl Acad Sci USA 88:8996-9000

Perez V, Roach B, Woods S, Srihari V, McGlashan T, Ford J, Mathalon D (2013) Early auditory gamma-band responses in patients at clinical high risk for schizophrenia. Suppl Clin Neurophysiol 62:147-162

Perrin F, Bertrand O, Pernier J (1987) Scalp current density mapping: value and estimation from potential data. IEEE Trans Biomed Eng 34:283-288

Pittman-Polletta BR, Kocsis B, Vijayan S, Whittington MA, Kopell $\mathrm{NJ}$ (2015) Brain rhythms connect impaired inhibition to altered cognition in schizophrenia. Biol Psychiatry 77:1020-1030

Reichenberg A, Harvey PD, Bowie CR, Mojtabai R, Rabinowitz J, Heaton RK, Bromet E (2009) Neuropsychological function and dysfunction in schizophrenia and psychotic affective disorders. Schizophr Bull 35:1022-1029

Ribary U (2005) Dynamics of thalamo-cortical network oscillations and human perception. Prog Brain Res 150:127-142

Ribary U, Ioannides AA, Singh KD, Hasson R, Bolton JP, Lado F, Mogilner A, Llinas R (1991) Magnetic field tomography of coherent thalamocortical $40-\mathrm{Hz}$ oscillations in humans. Proc Natl Acad Sci USA 88:11037-11041

Roach BJ, Mathalon DH (2008) Event-related EEG time-frequency analysis: an overview of measures and an analysis of early gamma band phase locking in schizophrenia. Schizophr Bull 34:907-926

Roach BJ, Ford JM, Hoffman RE, Mathalon DH (2013) Converging evidence for gamma synchrony deficits in schizophrenia. Suppl Clin Neurophysiol 62:163-180

Rothenberger A (2009) Brain oscillations forever-neurophysiology in future research of child psychiatric problems. J Child Psychol Psychiatry 50:79-86

Shenton ME, Dickey CC, Frumin M, McCarley RW (2001) A review of MRI findings in schizophrenia. Schizophr Res 49:1-52

Singer W (1999) Neuronal synchrony: a versatile code for the definition of relations? Neuron 24(49-65):111-125

Singer W (2001) Consciousness and the binding problem. Ann N Y Acad Sci 929:123-146

Singer W (2009) Distributed processing and temporal codes in neuronal networks. Cogn Neurodyn 3:189-196

Skåtun KC, Kaufmann T, Tønnesen S, Biele G, Melle I, Agartz I, Alnæs D, Andreassen OA, Westlye LT (2016) Global brain connectivity alterations in patients with schizophrenia and bipolar spectrum disorders. J Psychiatry Neurosci 41:331-341

Spencer KM, Nestor PG, Perlmutter R, Niznikiewicz MA, Klump MC, Frumin M, Shenton ME, McCarley RW (2004) Neural synchrony indexes disordered perception and cognition in schizophrenia. Proc Natl Acad Sci USA 101:17288-17293

Spencer KM, Niznikiewicz MA, Shenton ME, McCarley RW (2008) Sensory-evoked gamma oscillations in chronic schizophrenia. Biol Psychiatry 63:744-747

Stam CJ (2005) Nonlinear dynamical analysis of EEG and MEG: review of an emerging field. Clin Neurophysiol 116:2266-2301

Steriade M (2006) Grouping of brain rhythms in corticothalamic systems. Neuroscience 137:1087-1106

Symond MP, Harris AW, Gordon E, Williams LM (2005) "Gamma synchrony" in first-episode schizophrenia: a disorder of temporal connectivity? Am J Psychiatry 162:459-465

Uhlhaas PJ (2013) Dysconnectivity, large-scale networks and neuronal dynamics in schizophrenia. Curr Opin Neurobiol 23:283-290

Uhlhaas PJ, Singer W (2010) Abnormal neural oscillations and synchrony in schizophrenia. Nat Rev Neurosci 11:100-113

Uhlhaas PJ, Singer W (2011) The development of neural synchrony and large-scale cortical networks during adolescence: relevance for the pathophysiology of schizophrenia and neurodevelopmental hypothesis. Schizophr Bull 37:514-523

Uhlhaas PJ, Singer W (2012) Neuronal dynamics and neuropsychiatric disorders: toward a translational paradigm for dysfunctional large-scale networks. Neuron 75:963-980

Uhlhaas PJ, Haenschel C, Nikolić D, Singer W (2008) The role of oscillations and synchrony in cortical networks and their putative relevance for the pathophysiology of schizophrenia. Schizophr Bull 34:927-943

Uhlhaas PJ, Roux F, Rodriguez E, Rotarska-Jagiela A, Singer W (2010) Neural synchrony and the development of cortical networks. Trends Cogn Sci 14:72-80

Uhlhaas PJ, Roux F, Singer W (2013) Thalamocortical synchronization and cognition: implications for schizophrenia? Neuron 77:997-999 
Varela F, Lachaux JP, Rodriguez E, Martinerie J (2001) The brainweb: phase synchronization and large-scale integration. Nat Rev Neurosci 2:229-239

Ventura J, Green MF, Shaner A, Liberman RP (1993) Training and Quality Assurance with the Brief Psychiatric Rating-Scale - the Drift Busters. Int J Meth Psych Res 3:221-244

Ventura J, Nuechterlein KH, Subotnik KL, Gutkind D, Gilbert EA (2000) Symptom dimensions in recent-onset schizophrenia and mania: a principal components analysis of the 24-item Brief Psychiatric Rating Scale. Psychiatry Res 97:129-135

Wagner G, Koch K, Schachtzabel C, Schultz CC, Gaser C, Reichenbach JR, Sauer H, Bar KJ, Schlosser RG (2013) Structural basis of the fronto-thalamic disconnectivity in schizophrenia: A combined DCM-VBM study. Neuroimage Clin 3:95-105
Ward LM (2011) The thalamic dynamic core theory of conscious experience. Conscious Cogn 20:464-486

Welsh RC, Chen AC, Taylor SF (2010) Low-frequency BOLD fluctuations demonstrate altered thalamocortical connectivity in schizophrenia. Schizophr Bull 36:713-722

Woodward ND (2016) The course of neuropsychological impairment and brain structure abnormalities in psychotic disorders. Neurosci Res 102:39-46

Woodward ND, Karbasforoushan H, Heckers S (2012) Thalamocortical dysconnectivity in schizophrenia. Am J Psychiatry 169:1092-1099

Xing D, Shen Y, Burns S, Yeh CI, Shapley R, Li W (2012) Stochastic generation of gamma-band activity in primary visual cortex of awake and anesthetized monkeys. J Neurosci 32:13873a-13880a 